D. Boey $\cdot$ S. Lin · T. Karl • P. Baldock $\cdot$ N. Lee

R. Enriquez $\cdot$ M. Couzens $\cdot$ K. Slack $\cdot$ R. Dallmann •

A. Sainsbury $\cdot$ H. Herzog

\title{
Peptide $\mathrm{Y}$ ablation in mice leads to the development of hyperinsulinaemia and obesity
}

Received: 6 September 2005 / Accepted: 10 February 2006 / Published online: 21 April 2006

C) Springer-Verlag 2006

\begin{abstract}
Aims/hypothesis: Obese people exhibit reduced circulating peptide YY (PYY) levels, but it is unclear whether this is a consequence or cause of obesity. We therefore investigated the effect of Pyy ablation on energy homeostasis. Methods: Body composition, i.p. glucose tolerance, food intake and hypothalamic neuropeptide expression were determined in Pyy knock-out and wildtype mice on a normal or high-fat diet. Results: Pyy knock-out significantly increased bodyweight and increased fat mass by $50 \%$ in aged females on a normal diet. Male chow-fed $\mathrm{Pyy}^{--}$mice were resistant to obesity but became significantly fatter and glucose-intolerant compared with wild-types when fed a high-fat diet. Pyy knock-out animals exhibited significantly elevated fasting or glucose-stimulated serum insulin concentrations vs wild-types, with no increase in basal or fasting-induced food intake. Pyy knock-out decreased or had no effect on neuropeptide Y expression in the arcuate nucleus of the hypothalamus, and significantly increased proopiomelanocortin expression in this region. Male but not female
\end{abstract}

The last two authors named contributed equally to the work.

D. Boey $\cdot$ S. Lin $\cdot$ T. Karl $\cdot$ N. Lee $\cdot$ M. Couzens $\cdot$ K. Slack

A. Sainsbury $\cdot$ H. Herzog $(\bowtie)$

Neurobiology Research Programme,

Garvan Institute of Medical Research, St Vincent's Hospital,

384 Victoria Street, Darlinghust,

Sydney, NSW 2010, Australia

e-mail: h.herzog@garvan.org.au

Tel.: +61-29-2958296

Fax: +61-29-2958281

P. Baldock · R. Enriquez

Bone and Mineral Research Programme,

Garvan Institute of Medical Research, St Vincent's Hospital,

Sydney, NSW, Australia

R. Dallmann

Department of Zoology, University of Toronto,

Toronto, ON, Canada

T. Karl

Neuroscience Institute of Schizophrenia and Allied Disorders,

Sydney, NSW, Australia knock-outs exhibited significantly increased growth hormone-releasing hormone expression in the ventromedial hypothalamus and significantly elevated serum IGF-I and testosterone levels. This sex difference in activation of the hypothalamo-pituitary somatotrophic axis by $P y y$ ablation may contribute to the resistance of chow-fed male knockouts to late-onset obesity. Conclusions/interpretation: PYY signalling is important in the regulation of energy balance and glucose homeostasis, possibly via regulation of insulin release. Therefore reduced PYY levels may predispose to the development of obesity, particularly with ageing or under conditions of high-fat feeding.

Keywords Diabetes $\cdot$ Hyperinsulinaemia Obesity Peptide YY

Abbreviations DXA: dual-energy X-ray absorptiometry . GHRH: growth hormone-releasing hormone $\cdot$ NPY: neuropeptide Y P POMC: proopiomelanocortin - PPY: pancreatic polypeptide $\cdot$ PYY: peptide YY

\section{Introduction}

The gut hormone peptide YY (PYY) belongs to the neuropeptide Y (NPY) family along with pancreatic polypeptide (PPY) [1]. PYY, PPY and NPY mediate their effects through $\mathrm{Y}$ receptors, of which there are several types (Y1, Y2, Y4, Y5 and Y6) [2]. The endocrine L cells of the lower gastrointestinal tract are the major source of PYY; however, PYY is also produced in the alpha cells of the islets of Langerhans as well as in the stomach and the brainstem [3].

There are two endogenous forms of PYY: PYY1-36 and PYY3-36. The latter is produced by the action of the cellsurface enzyme dipeptidyl peptidase IV on secreted PYY136 [4]. PYY1-36 binds to all known Y receptors, albeit with differing affinities, whereas PYY3-36 is more selective for the Y2 receptor and to a lesser extent to the Y5 receptor [5]. Upon ingestion of a meal, PYY levels rise within $15 \mathrm{~min}$, peak at $60 \mathrm{~min}$ and remain elevated for up to 
$6 \mathrm{~h}$ in humans [6]. The initial rise of PYY appears to be due to an indirect neural reflex, whereas the sustained release of PYY appears to be a direct effect of intraluminal contents on endocrine L cells [7]. Dietary fat is the most potent stimulant of PYY release [6].

Both PYY1-36 [8, 9] and PYY3-36 suppress appetite and food intake in rodents [10-14]. However, one report was unable to confirm this finding [15]. Most studies investigating the role of PYY in energy balance have focused primarily on its effects on feeding and bodyweight, and less is known about the role of PYY in influencing body composition and hormonal and metabolic parameters that influence it, such as leptin etc. Indeed, many factors that play a significant role in regulating energy homeostasis do so in the absence of corresponding effects on food intake or bodyweight $[16,17]$. There is evidence that PYY could influence energy balance via multiple pathways aside from effects on food intake. Notably, PYY has been shown to slow the transit of food through the gastrointestinal tract, delay gastric and gallbladder emptying $[6,18]$ and delay pancreatic and intestinal secretion [19] via direct actions on target tissues or indirect actions via vagal pathways [7].

It has also been implied that PYY has an important function in regulating insulin release and glucose homeostasis. Previous in vitro studies demonstrate that PYY acts to inhibit insulin secretion and glucose-stimulated insulin release from isolated pancreatic islets [20, 21]. In obese rodent models PYY3-36 reinforces insulin action on glucose disposal independently of changes in food intake and bodyweight $[14,22]$. Since changes in gastrointestinal function and circulating insulin concentrations per se have been shown to have profound effects on energy balance [23-25], it is possible that PYY may influence long-term energy balance via these mechanisms.

The aim of this work was to elucidate the role of PYY in the regulation of multiple hormonal and metabolic determinants of lean and fat mass. To this end, we generated Pyy knock-out mice and studied them under a range of conditions that are known to influence energy homeostasis, notably fasting, ageing and a high-fat diet.

\section{Material and methods}

Animal care

All research and animal care procedures were approved by the Garvan Institute/St Vincent's Hospital Animal Experimentation Ethics Committee.

Pyy targeting vector construction and gene disruption

The $130 \mathrm{~kb}$ mouse genomic BAC clone was mapped and various fragments were subcloned. A $10.5 \mathrm{~kb}$ SpeI fragment containing $6 \mathrm{~kb} 5^{\prime}$-flanking sequence, the entire Pyy gene and a $3 \mathrm{~kb}$ 3'-flanking sequence was chosen for the construction of a Pyy-Cre knock-in construct. The linearised version of that clone was transfected into ES cells. Two positive clones were injected into C57BL/6 blastocysts and chimeric mice were bred to generate heterozygous mice, and subsequently homozygous Pyy-Cre knock-in mice were bred. The genotype of the mice were determined by Southern blot analysis of NheI-digested DNA employing probes located outside the targeting sequence. Probe A and B were generated by PCR (A for: 5'-AGTGATTTGCTCA GAAGC-3' and rev: 5'-CTAGTTCTATAGACCAGAC-3') and (B for: $5^{\prime}$-CTGCCATGGCTGACCATGC-3' and B rev: $5^{\prime}$-TGGT GGTGGCATGCACAC-3'). The conditions for all PCR reactions were 35 cycles of $94^{\circ} \mathrm{C}$ for $45 \mathrm{~s}, 58^{\circ} \mathrm{C}$ for $1 \mathrm{~min}$ and $72^{\circ} \mathrm{C}$ for $20 \mathrm{~s}$.

\section{Measurement of food intake and bodyweight}

Wild-type and $P y y^{-/}$animals originating from three or four different breeding pairs were housed under conditions of controlled temperature $\left(22^{\circ} \mathrm{C}\right)$ and lighting (12-h light cycle, lights on at $07.00 \mathrm{~h}$ ). Male and female wild-type and $P y y^{-1-}$ mice had free access to a normal chow diet (6\% of calories from fat, $21 \%$ from protein, $71 \%$ from carbohydrate; $2.6 \mathrm{kcal} / \mathrm{g}$; Gordon's Speciality Stock Feeds, Yanderra, NSW, Australia). Separate groups of wild-type and $\mathrm{Pyy}^{-1-}$ mice were fed a high-fat diet $(46 \%$ of calories from fat, $21 \%$ from protein, $33 \%$ from carbohydrate; $4.72 \mathrm{kcal} / \mathrm{g}$ ) from 4 to 5 weeks of age onwards. The high-fat diet, made in-house, was based on the composition of Rodent Diet Catalogue Number D12451 (Research Diets, New Brunswick, NJ, USA), with the exception that safflower oil and copha were used in place of soybean oil and lard, respectively. Bodyweight was monitored weekly from 4 to 28 weeks of age for the chow-fed animals and 4 to 14 weeks of age for the highfat-fed animals. At 11 to 12 weeks of age, basal and fasting-induced food and water intake were measured. Basal food and water intake were also determined at 24 weeks of age in male and female $P y y^{--}$and control mice.

\section{Glucose tolerance tests}

At 13 weeks of age, chow-fed and high-fat-fed wild-type and Pyy knock-out mice were fasted for $24 \mathrm{~h}$ prior to i.p. glucose tolerance tests $(1 \mathrm{~g} / \mathrm{kg})$. Serial blood samples were collected from the tail for determination of serum glucose and insulin levels. Glucose AUCs were calculated between 0 and $120 \mathrm{~min}$ after glucose injection and expressed as $\mathrm{mmol} \cdot 1^{-1} \cdot \mathrm{min}^{-1}$. Insulin AUCs were calculated between 0 and $120 \mathrm{~min}$ after glucose injection and expressed as $\mathrm{pmol} \cdot \mathrm{l}^{-1} \cdot \mathrm{min}^{-1} \cdot \mathrm{g}$ bodyweight ${ }^{-1}$.

\section{Tissue collection and analysis}

Wild-type and $\mathrm{Pyy}^{--}$mice at 14 or 28 weeks of age were killed by cervical dislocation between 12.00 and $14.00 \mathrm{~h}$ for collection of trunk blood. Brains were immediately 
removed and frozen on dry ice. Mice were scanned for whole-body lean and fat mass using a dual-energy X-ray absorptiometry (DXA) (Lunar PIXImus2 mouse densitometer; GE Healthcare Technologies, Waukesha, WI, USA). White adipose tissue depots (right inguinal, right epididymal or periovarian [gonadal], right retroperitoneal and mesenteric) were removed and weighed. Serum glucagon levels were measured by RIA kits from Linco Research (St Louis, MO, USA), insulin levels were measured using an ELISA kit from Mercodia (Uppsala, Sweden), serum-free $\mathrm{T} 4$ and testosterone concentrations were measured with kits from ICN Biomedicals (Costa Mesa, CA, USA), serum IGF-I was determined using an RIA kit from Bioclone (Marrickville, NSW, Australia), serum glucose was determined with a glucose oxidase assay kit (Trace Scientific, Melbourne, VIC, Australia), and serum triglyceride and NEFAs were determined with kits from Roche Diagnostics (Mannheim, Germany) and Wako (Osaka, Japan), respectively.

PYY, glucagon and PPY staining

Pancreas and segments of the small intestine and colon obtained from three wild-type and three knock-out animals were fixed in $4 \%$ paraformaldehyde in PBS, processed, embedded in paraffin and cut at $7 \mu \mathrm{m}$ sections. Sections were deparaffinised, rehydrated and incubated in $1 \% \mathrm{H}_{2} \mathrm{O}_{2}$ in methanol for $20 \mathrm{~min}$. Sections were then rinsed in PBS and blocked with either $20 \%$ normal goat serum or donkey serum in PBS for $20 \mathrm{~min}$. Rabbit anti-human PYY antiserum $(1: 1,000)$ (Peninsula Laboratories, CA, USA), rabbit anti-glucagon antibody (1:500) (ICN), or guinea pig anti-rat PPY serum (Linco Research) $(1: 1,000)$ was applied for $1 \mathrm{~h}$ at room temperature. For the PYY antibody, crossreactivity was minimised by incubating the antiserum with protein extracted from brain of $P y y^{--}$mice. Slides were rinsed in PBS before incubation with a peroxidaseconjugated goat anti-rabbit $\operatorname{IgG}(\mathrm{H}+\mathrm{L})$ antibody $(1: 1,000)$ (Zymed Laboratories, San Francisco, CA, USA) or a biotinylated donkey anti-guinea-pig IgG antibody $(1: 5,000)$ (Jackson ImmunoResearch Laboratories, West Grove, PA, USA) for $30 \mathrm{~min}$ at room temperature. The sections incubated with the biotinylated antibody were rinsed in PBS and incubated with Avidin-Biotin-Peroxidase Vectastain (Vector Laboratories, Burlingame, CA, USA) for $30 \mathrm{~min}$ at room temperature. Sections were washed in PBS and treated with diaminobenzidine from DAKO (Carpinteria, CA, USA) for $5 \mathrm{~min}$. Slides were rinsed in water, counterstained with haematoxylin and dehydrated through to xylene before coverslipping.

\section{Measurement of islet cell size and number}

Six random pancreatic sections were collected from five wild-type and five $P y y^{-/}$male mice. H\&E stained sections were observed using a ProgRes 3008 camera (Zeiss, Jena, Germany) mounted on a Zeiss Axiophot microscope. The average number and size of islet cells per section were quantified using the $\times 20$ objective and Leica IM 1000 version 1.20 software (Leica Microsystems, Heerbrig, Switzerland).

\section{In situ hybridisation}

Coronal brain sections $(20 \mu \mathrm{m})$ were cut on a cryostat and thaw-mounted on Superfrost slides (Menzel-Glaser, Brunswick, Germany). Matching sections from the same coronal brain level of $P y y$ knock-out and wild-type mice ( $n=5$ mice/ group) were assayed together using DNA oligonucleotides complementary to mouse Npy (5'-GAGGGTCAGTCCA CACAGCCCCATTCGCTTGTTACCTAGCAT-3'); mouse proopiomelanocortin (Pomc) (5'-TGGCTGCTCTCCAGG CACCAGCTCCACACATCTATGGAGG- $\left.3^{\prime}\right)$; and mouse growth hormone-releasing hormone $(G h r h)\left(5^{\prime}\right.$-GCTTGT CCTCTGTCCACATGCTGTCTTCCTGGCGGCTYGAG CCTGG-3') as described previously [26].

For evaluation of mRNA levels in scattered neurons, images from dipped sections were digitised using a ProgRes 3008 camera (Zeiss) mounted on a Zeiss Axiophot microscope. Silver grain density over single neurons was evaluated using NIH-Image 1.61 software (written by Wayne Rasband and available from anonymous FTP at zippy.nimh.nih.gov). Background labelling was uniform and never exceeded $5 \%$ of specific signal.

\section{Activity measurements}

The total cage activity was recorded using a passive infrared detector (PID) (Conrad Electronics, Hirschau, Germany) on top of the cage lid of single-housed 16-weekold chow-fed animals. The signals of the PIDs were detected by an I/O interface card (PIO48 II; Dr Schetter BMC Ing, Puchheim, Germany) and stored using custommade software. To show the activity pattern of the mice, data were plotted so that each cycle's activity is shown both to the right and below that of the previous cycle, a so-called double-plotted actogram with the $x$-axis showing $48 \mathrm{~h}$ starting on the left with $00.00 \mathrm{~h}$ (ZT 6). In order to test for differences in total activity between the genotypes, the activity counts were summed up to 30 -min bins and a mean/day was calculated by averaging corresponding bins of seven consecutive days.

\section{Statistical analyses}

Results for bodyweight, lean and fat mass, food intake, activity measurements and differences in serum glucose and insulin levels during glucose tolerance tests were compared among groups using repeated-measures ANOVA followed by Fisher's post hoc tests. Differences in serum hormone and metabolite concentrations between $\mathrm{Pyy}^{-1}$ and wild-type mice were assessed by ANOVA with subsequent Fisher's post hoc tests. Alterations in neuro- 
peptide mRNA expression and pancreatic islet parameters between knock-out and wild-type mice were assessed by ANOVA. StatView version 4.5 (Abacus Concepts, Berkeley, CA, USA) was used for all statistical analyses and $p<0.05$ was accepted as being statistically significant.

\section{Results}

Generation of Pyy knock-out mice

A knock-out-targeting vector for the $P y y$ gene was designed which also allows the Pyy promoter-driven expression of the Cre-recombinase gene under control of the inducible tetracycline promoter (Fig. 1a). Two positive ES cell clones for the Pyy construct were used to obtain chimeric mice, heterozygotes, and subsequently homozygous knock-out mice. DNA isolated from tail tips was used in Southern blot analysis (NheI) to confirm correct integration and modification of the targeted allele (Fig. 1b,c).

Breeding of heterozygous germline knock-out animals produced a slight deviation from the expected Mendelian ratio of genotypes of 25:50:25 to 43:38:19 (wild-types: heterozygotes:homozygous knock-outs, $n=7$ breeding pairs). However, $\mathrm{Pyy}^{--}$mice breed normally with an average litter size of $7.15 \pm 0.37$ pups/litter, which was not significantly different from wild-type litter sizes of $6.57 \pm$ 0.97 pups $(n=7-13$ breeding pairs). The gender ratio in $\mathrm{Pyy}^{--}$offspring shows a trend towards a greater proportion of females $(57.1 \%, n=112)$.

Fig. 1 Generation of $\mathrm{Pyy}^{-1}$ mice. a Targeting vector design and screening strategy. The bold horizontal bars indicate the position of probes used for Southern analysis of genomic DNA from targeted ES cells as well as knock-out animals. b, c Southern analysis of genomic DNA isolated from wild-type $(+/+)$, heterozygous $(+/-)$ and knock-out $(-/-)$ mice cut with NheI or $B a m H I$, using probes $\mathrm{A}$ and $\mathrm{B}$ (see Material and methods, Pyy targeting vector construction...), respectively
Unaltered tissue morphology in $P y y^{-/}$mice

Successful deletion of the Pyy gene was confirmed by the absence of staining for PYY in the pancreas and colon of $P y y^{-/}$mice (Fig. 2a-d). The morphology of these PYYexpressing tissues did not show any obvious changes when examined using H\&E staining. Moreover, the average size of the islets of Langerhans in knock-out mice $(0.013 \pm$ $\left.0.0002 \mathrm{~mm}^{2}\right)$ did not differ significantly from that of wildtype mice $\left(0.020 \pm 0.0005 \mathrm{~mm}^{2}\right)(n=5$ mice/genotype). The same is true for the number of islets per section $(8.2 \pm 1.5$ in wild-type mice vs $6.6 \pm 1.3$ in $\mathrm{Pyy}^{--}$mice, six sections from five mice of each genotype). Importantly, the expression of the Ppy gene, which is located only $7 \mathrm{~kb}$ downstream of the Pyy gene locus, was not influenced by the removal of the Pyy gene (Fig. 2e,f), nor was the expression of glucagon (Fig. 2g,h) or insulin (data not shown) in the islets of Langerhans.

Effects of Pyy deletion on bodyweight, adiposity and food intake

No change in bodyweight was observed in male $P y y^{-/}$ mice between 4 and 28 weeks of age under chow-fed conditions (Fig. 3a,b), which was also confirmed by the comparison of body-weight AUC measurements (wildtype $628.5 \pm 7.8 \mathrm{~g} / 24$ weeks vs $P y y^{-/} 657.1 \pm 21.3 \mathrm{~g} / 24$ weeks) $(p=0.2)$. However, female $P y y^{-/}$mice had a significantly higher bodyweight than controls under chowfed conditions from 4 to 28 weeks of age (Fig. 3a) (AUC:

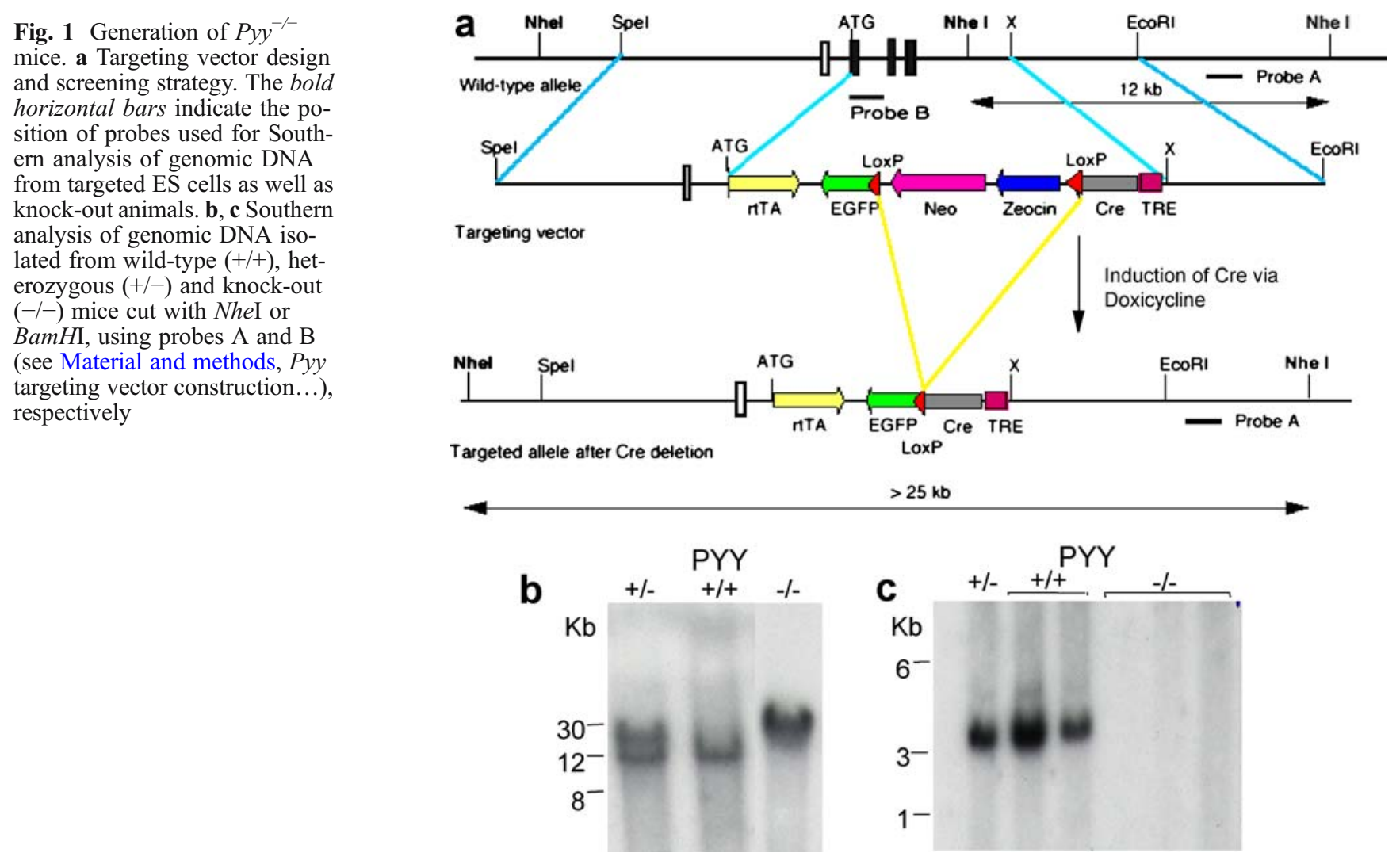




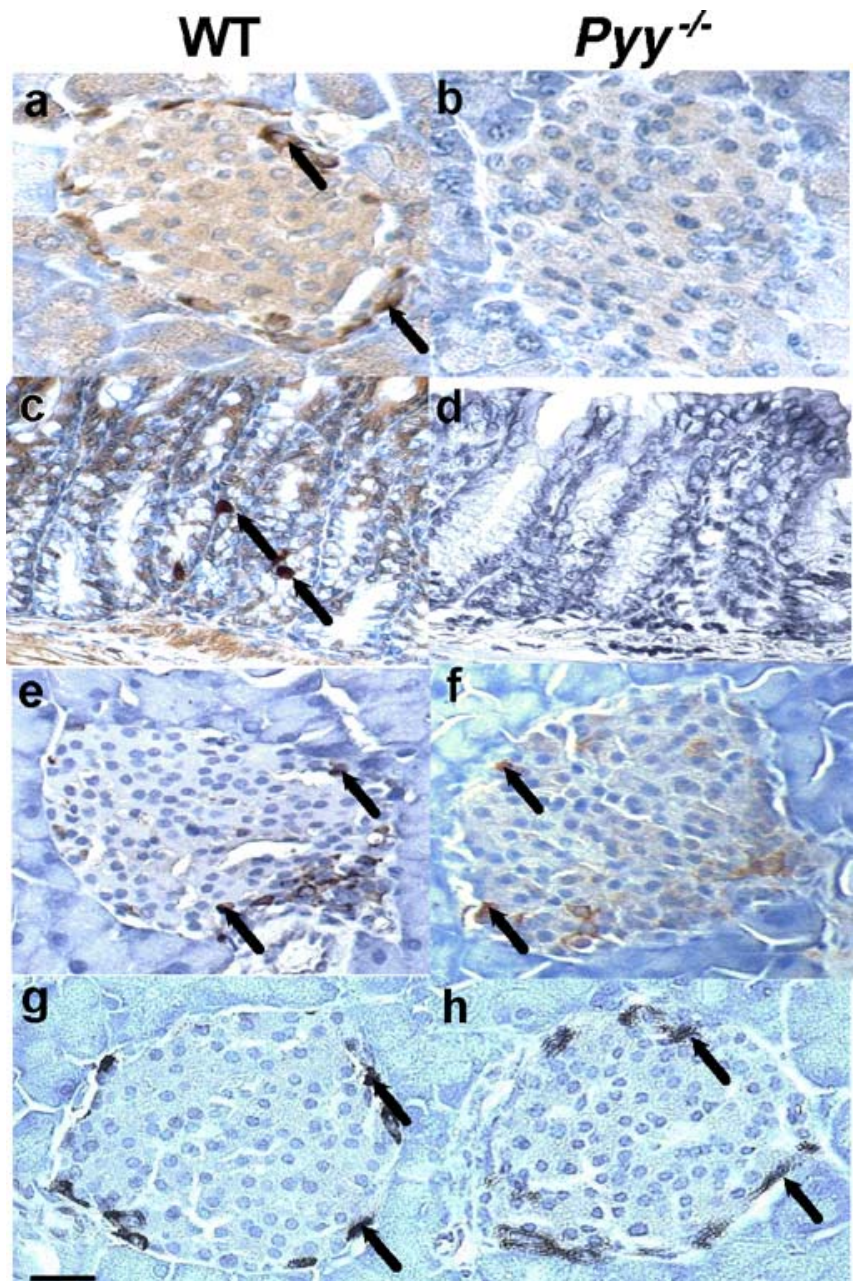

Fig. 2 Immunostaining for PYY, PPY and glucagon. PYY staining in the islets of Langerhans and colon of wild-type mice $(\mathbf{a}, \mathbf{c})$ and $P y y^{-/}$mice $(\mathbf{b}, \mathbf{d})$. e, $\mathbf{f}$ PPY staining in pancreas of $P y y^{-/}$and wildtype mice, respectively. $\mathbf{g}, \mathbf{h}$ Glucagon staining in pancreas of $P y y^{--}$ and wild-type mice, respectively. Arrows indicate the positively stained cells. Scale bar $=10 \mu \mathrm{m}$. Pictures are representative of staining observed in tissues obtained from 14-week-old animals ( $n=3 \mathrm{mice} /$ group)

wild-type $505.0 \pm 8.9 \mathrm{~g} / 24$ weeks; $P y y^{-/-} 554.0 \pm 14.5 \mathrm{~g} / 24$ weeks) $(p<0.01)$. Both female and male 14-week-old chow-fed $\mathrm{Pyy}^{-/}$mice had a significant increase in total body lean mass (Fig. 3d,f) with the females also showing a significant decrease in whole body fat mass at 14 weeks as determined by DXA (Fig. 2e). However, female $\mathrm{Pyy}^{-/}$ mice exhibited a marked increase in bodyweight gain from

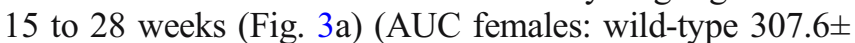
$7.3 \mathrm{~g} / 15-28$ weeks; $P y y^{--} 344.0 \pm 10.4 \mathrm{~g} / 15-28$ weeks) $(p<0.02)$, which was not observed in the male knock-outs (Fig. 3b) (AUC males: wild-type 381.8 $\pm 6 . \mathrm{g} / 15-28$ weeks; $P y y^{--} 401.0 \pm 16.3 \mathrm{~g} / 15-28$ weeks $)(p=0.23)$. Consistent with the increased bodyweight gain, chow-fed female $P y y^{--}$ mice showed a significant $50 \%$ increase in fat mass at 28 weeks of age as determined by DXA (Fig. 3e). This was also confirmed by marked and significant increases in the weight of all white adipose tissue depots measured in female chow-fed $\mathrm{Pyy}^{--}$mice at 28 weeks of age (Table 1).
These findings show that female but not male $P y y^{-/}$ mice develop late-onset obesity, as indicated by significant increases in bodyweight and fat mass when fed a chow diet. It is well documented that long-term exposure to high-fat diet causes obesity in a number of species [27]. Moreover, PYY release from the gut is strongly stimulated by dietary fat [7]. Considering male knock-outs did not develop lateonset obesity, we were interested in determining whether high-fat feeding would induce obesity similarly to that observed in female knock-outs. Therefore we investigated the effect of a high-fat diet on bodyweight and energy homeostasis in male $\mathrm{Pyy}^{--}$and wild-type mice. When fed a high-fat diet from 4 weeks of age onwards, male $\mathrm{Pyy}^{-/}$ mice showed no significant change in bodyweight when compared with wild-type mice (Fig. 3c). However, these high-fat-fed $P y y^{-1}$ mice showed a significant increase in total body fat mass when compared with wild-types, as determined by DXA (Fig. 3g), as well as a significant increase in the weight of the gonadal white adipose tissue depot (Table 2).

In young chow-fed female mice, the observed changes in body composition (increased lean mass and decreased fat mass) were associated with a significant decrease in nonfasted food and water intake (Table 1). Interestingly, however, the increases in bodyweight and/or fat mass observed in older female and high-fat-fed male $\mathrm{Pyy}^{-/-}$mice were not associated with any significant changes in nonfasted food or water intake (Tables 1 and 2). Additionally, there was no significant difference between 14-week-old chow- or fat-fed $P y y^{--}$and wild-type mice with respect to food intake at 1, 2, 8, 24, 48 or $72 \mathrm{~h}$ after a 24 -h fast (data not shown).

\section{Effect of Pyy deficiency on glucose homeostasis}

There was no significant difference between wild-type and $P y y^{-/}$mice with respect to fed serum glucose levels (data not shown). When animals were fasted for $24 \mathrm{~h}$ prior to the glucose tolerance test at 13 weeks of age, there was no significant difference between wild-type and knock-out mice with regards to basal serum glucose levels, independently of dietary condition (Fig. 4a-c). There was no effect of $P y y$ knock-out on glucose tolerance in female or male chow-fed mice at 13 weeks of age (Fig. 4a,d and b,e). Interestingly, both male and female chow-fed $\mathrm{Pyy}^{--}$mice showed significantly higher serum insulin levels than controls throughout the course of the glucose tolerance tests (Fig. 4g,j and h, $\mathrm{k})$. As weight gain is known to drive insulin resistance, insulin levels during the glucose tolerance test and the insulin AUC were calculated by normalising for bodyweight. This adjustment of the data did not change the shape of the curves shown in Fig. 4g,h (data not shown), nor did it change the AUC for insulin for females (wildtype $458.7 \pm 68.1 \mathrm{pmol} \cdot 1^{-1} \cdot \mathrm{min}^{-1} \cdot \mathrm{g}^{-1}$ vs $P y y^{-/} 659.8 \pm$ $\left.62.8 \mathrm{pmol} \cdot \mathrm{l}^{-1} \cdot \mathrm{min}^{-1} \cdot \mathrm{g}^{-1}\right)(p<0.05)$ and males (wildtype $338.7 \pm 32.6 \mathrm{pmol} \cdot 1^{-1} \cdot \mathrm{min}^{-1} \cdot \mathrm{g}^{-1}$ vs $P y y^{-/} 442.4 \pm$ $\left.27.7 \mathrm{pmol} \cdot 1^{-1} \cdot \min ^{-1} \cdot \mathrm{g}^{-1}\right)(p<0.04)$. 
a

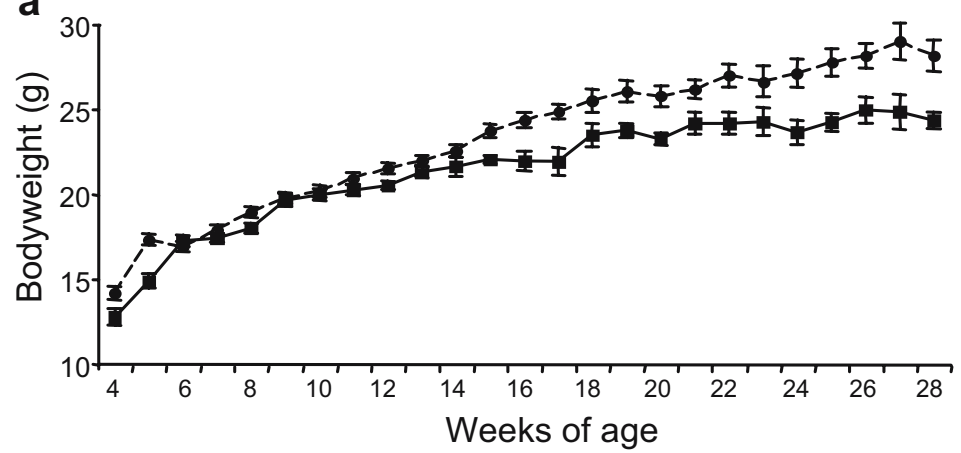

b

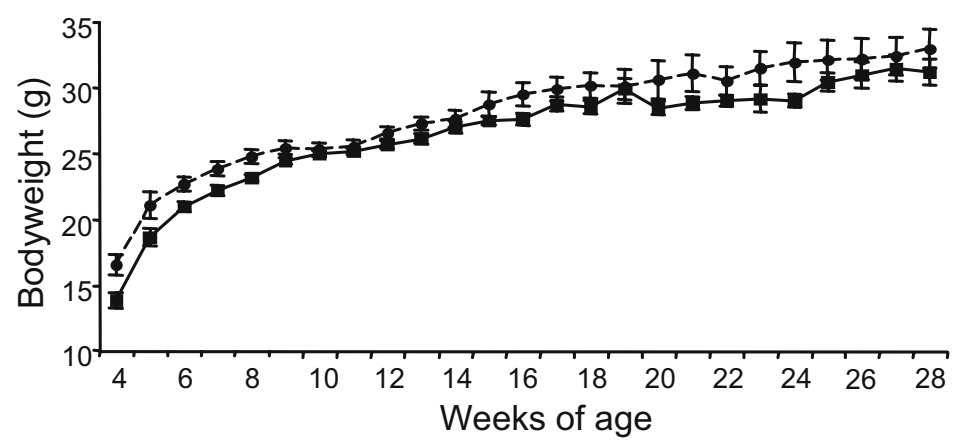

C

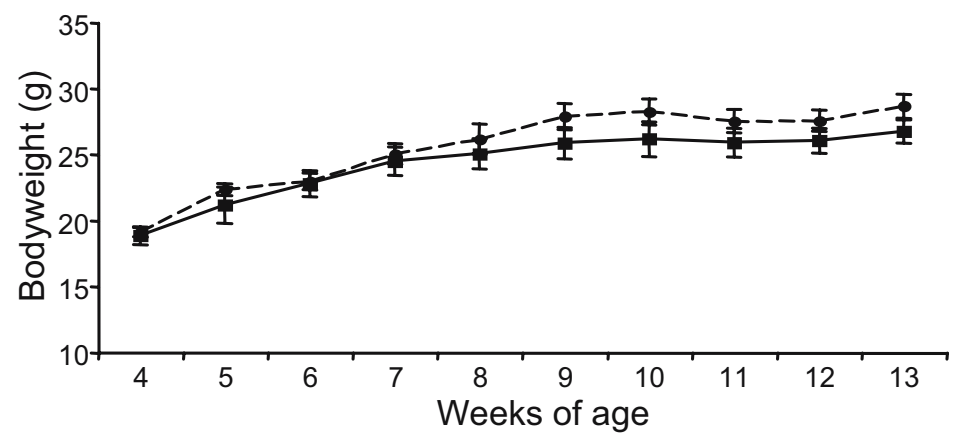

d

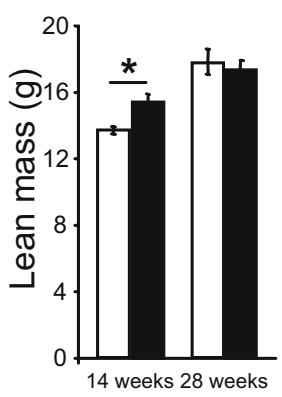

e

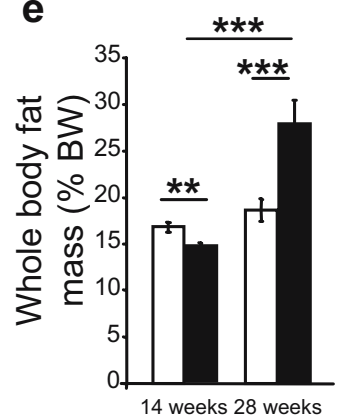

f

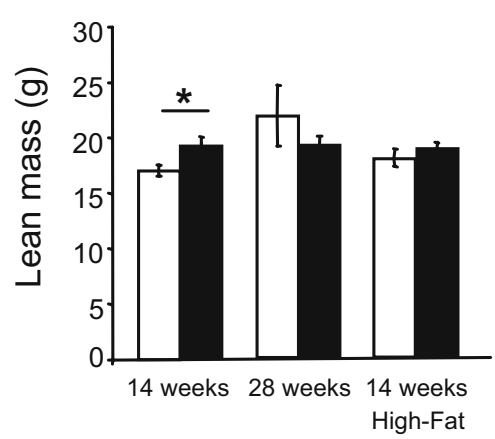

g

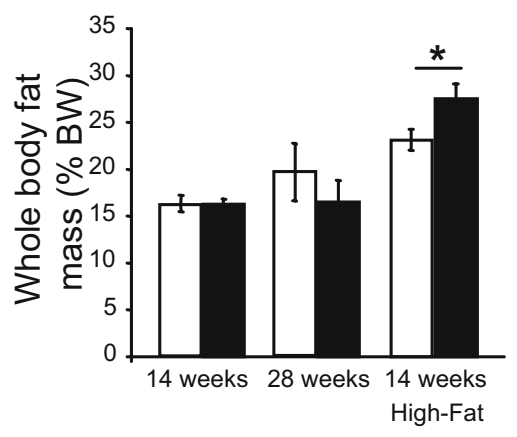

Fig. 3 Effect of PYY deficiency on bodyweight $(\mathbf{a}-\mathbf{c})$, lean mass $(\mathbf{d}, \mathbf{f})$ and adiposity $(\mathbf{e}, \mathbf{g})$. a-c Bodyweight curves from 4 to 28 weeks of age for a female (wild-type $n=37 ; P y y^{-1} n=35$ ) and $\mathbf{b}$ male (wild-type $n=32$; $P y y^{-/} n=33$ ) wild-type (filled squares) and $P_{y y}{ }^{-1}$ (filled circles) mice fed a chow diet. c Bodyweight curves for high-fat-fed male wild-type (filled squares) $(n=8)$ and $P y y^{-1-}$ (filled circles) $(n=8)$ mice from 4 to 13 weeks of age. d, e Whole-body lean and fat mass obtained using DXA for female wild-type (open bars) and $P y y^{--}$(filled bars) mice on

Under conditions of high-fat feeding, male knock-outs were less glucose-tolerant than wild-type mice $(p<0.05)$, shown by a significant increase in the AUC (Fig. 4c,f). However, this difference did not persist when the data were normalised for bodyweight (wild-type $54.5 \pm 2.2 \mathrm{mmol} \cdot 1^{-1}$. $\left.\min ^{-1} \cdot \mathrm{g}^{-1} ; P y y^{-1-} 57.4 \pm 1.8 \mathrm{mmol} \cdot 1^{-1} \cdot \mathrm{min}^{-1} \cdot \mathrm{g}^{-1}\right)(p=0.33)$ Fasting and glucose-induced serum insulin levels are significantly elevated in Pyy knock-outs compared with wild-type mice fed a high-fat diet, whether expressed as absolute values (Fig. 4i,1) or normalised for bodyweight (wild-type $612.3 \pm 30.8 \mathrm{pmol} \cdot \mathrm{l}^{-1} \cdot \mathrm{min}^{-1} \cdot \mathrm{g}^{-1} ; \mathrm{Pyy}^{-/} 782.7 \pm$ $\left.59.3 \mathrm{pmol} \cdot 1^{-1} \cdot \min ^{-1} \cdot \mathrm{g}^{-1}\right)(p<0.02)$. a chow diet at 14 weeks (wild-type $n=7 ; P y y^{-/} n=8$ ) and 28 weeks of age (wild-type $\left.n=6 ; P y y^{-/-} n=8\right)$. f, $\mathbf{g}$ DXA results for male mice at 14 weeks (wild-type $n=8 ; P y y^{-} n=8$ ) and 28 weeks of age (wild-type $\left.n=7 ; P y y^{-(} n=7\right)$ on a chow diet and at 14 weeks of age on a high-fat diet (wild-type $n=8 ; P y y^{--} n=9$ ). Data are means \pm SEM of the number of mice shown in parentheses. ${ }^{*} p<0.05,{ }^{* *} p<0.01, * * * p<0.001$ vs wildtype mice. $B W$, bodyweight

Effect of Pyy knock-out on serum hormone concentrations

Since Pyy deletion significantly increases lean mass in young, chow-fed animals (Fig. 3d,f), we investigated serum concentrations of IGF-I, the main mediator of the growth effects of growth hormone. No changes were observed in IGF-I levels in the female knock-outs (Table 1). However, serum IGF-I levels were significantly increased in male $P y y^{-1-}$ mice under chow-fed but not highfat-fed conditions (Tables 1 and 2), consistent with the pattern of change in total body lean mass (Fig. 3f). 
Table 1 The effect of PYY deficiency on food and water intake, white adipose tissue (WAT) weights and serum parameters of 14- and 28-week-old chow-fed wild-type $(W T)$ and $\mathrm{Pyy}^{-/-}$mice

\section{WT}

Food intake (g/24 h) (11 weeks)

Female

Male

Water intake (g/24 h) (11 weeks)

Female

Male

WAT inguinal $(\mathrm{g})(14$ weeks $)$

Female

Male

WAT gonadal (g) (14 weeks)

Female

Male

WAT mesenteric $(\mathrm{g})$ (14 weeks)

Female

Male

WAT retroperitoneal $(\mathrm{g})$ (14 weeks)

Female

Male

Food intake (g/24 h) (24 weeks)

Female

Male

Water intake (g/24 h) (24 weeks)

Female

Male

WAT inguinal (g) (28 weeks)

Female

Male

WAT gonadal (g) (28 weeks)

Female

Male

WAT mesenteric (g) (28 weeks)

Female

Male

WAT retroperitoneal (g) (28 weeks)

Female

Male

IGF-I (nmol/1)

Female

Male

Testosterone (nmol/l) (male)

Glucagon (pmol/l)

Female

Male

Free T4 (pmol/l)

Female

Male

NEFA (mmol/1)

Female

Male

Triglycerides (mmol/l)

Female

Male
$7.71 \pm 0.22(12)$

$7.61 \pm 0.82(10)$

$4.66 \pm 0.34$ (12)

$5.03 \pm 0.41(10)$

$0.14 \pm 0.01(22)$

$0.13 \pm 0.01$ (18)

$0.13 \pm 0.02(22)$

$0.16 \pm 0.01$ (18)

$0.16 \pm 0.01(22)$

$0.18 \pm 0.02$ (18)

$0.02 \pm 0.003(22)$

$0.04 \pm 0.004$ (18)

$4.10 \pm 0.475$ (5)

$4.43 \pm 0.18$ (5)

$4.27 \pm 0.34$ (5)

$4.03 \pm 0.24$ (5)

$0.16 \pm 0.01(15)$

$0.22 \pm 0.01$ (14)

$0.20 \pm 0.04$ (15)

$0.29 \pm 0.03$ (14)

$0.18 \pm 0.02(15)$

$0.29 \pm 0.02$ (14)

$0.03 \pm 0.01(15)$

$0.08 \pm 0.01$ (14)

$37.9 \pm 2.88(10)$

$24.0 \pm 3.78$ (16)

$1.96 \pm 0.75$ (11)

$1.9 \pm 0.3(8)$

$9.3 \pm 2.3(10)$

$23.87 \pm 1.24(10)$

$18.04 \pm 1.18$ (11)

$1.04 \pm 0.031(9)$

$1.36 \pm 0.10$ (15)

$0.67 \pm 0.1(8)$

$1.0 \pm 0.1(15)$
$P y y^{-1}$

$4.73 \pm 0.43(9)^{*}$

$6.86 \pm 0.77$ (10)

$3.76 \pm 0.17(9)^{*}$

$3.75 \pm 0.10(10) *$

$0.11 \pm 0.005(22) * *$

$0.14 \pm 0.01$ (22)

$0.12 \pm 0.01$ (22)

$0.15 \pm 0.01$ (22)

$0.15 \pm 0.01$ (22)

$0.20 \pm 0.01$ (22)

$0.03 \pm 0.002(22)$

$0.04 \pm 0.004$ (22)

$3.73 \pm 0.30(5)$

$4.61 \pm 0.25$ (5)

$4.34 \pm 0.31$ (5)

$3.92 \pm 0.23$ (5)

$0.30 \pm 0.04(13)^{* *}$

$0.24 \pm 0.05$ (11)

$0.52 \pm 0.07(13)^{* *}$

$0.30 \pm 0.05$ (11)

$0.37 \pm 0.05(13)^{* *}$

$0.30 \pm 0.05$ (11)

$0.08 \pm 0.01(13)^{* *}$

$0.08 \pm 0.02$ (11)

$39.76 \pm 1.58$ (23)

$33.19 \pm 2.29(24)^{*}$

$20.80 \pm 6.86(13)^{*}$

$2.6 \pm 0.5$ (12)

$7.3 \pm 3.4$ (13)

$26.94 \pm 1.77$ (14)

$19.46 \pm 1.68(13)$

$1.23 \pm 0.06(14)^{*}$

$1.37 \pm 0.1(13)$

$1.02 \pm 0.1(12)^{*}$

$1.26 \pm 0.1(13)$

Data are means \pm SEM (number of mice in parentheses)

$* p<0.05, * * p<0.01$ vs WT mice 
Table 2 The effect of PYY deficiency on food and water intake, white adipose tissue (WAT) weights and serum parameters of 14-week-old high-fat-fed wild-type $(W T)$ and $P y y^{-/-}$mice

\begin{tabular}{lcc}
\hline & WT & $P^{-l-}$ \\
\hline Food intake $(\mathrm{g} / 24 \mathrm{~h})$ & $2.97 \pm 0.16(8)$ & $2.97 \pm 0.50(8)$ \\
Water intake $(\mathrm{g} / 24 \mathrm{~h})$ & $3.35 \pm 0.13(8)$ & $3.38 \pm 0.31(8)$ \\
WAT inguinal $(\mathrm{g})$ & $0.27 \pm 0.03(8)$ & $0.33 \pm 0.02(8)$ \\
WAT gonadal $(\mathrm{g})$ & $0.37 \pm 0.04(8)$ & $0.53 \pm 0.05(8)^{* *}$ \\
WAT mesenteric $(\mathrm{g})$ & $0.32 \pm 0.02(8)$ & $0.43 \pm 0.04(8)$ \\
WAT retroperitoneal $(\mathrm{g})$ & $0.12 \pm 0.02(8)$ & $0.14 \pm 0.01(8)$ \\
IGF-I (nmol/l) & $33.31 \pm 1.96(8)$ & $29.47 \pm 5.36(9)$ \\
Testosterone (nmol/l) & $3.47 \pm 1.07(13)$ & $18.40 \pm 7.15(8)$ \\
Free T4 (pmol/l) & $16.18 \pm 1.32(7)$ & $16.21 \pm 1.43(12)$ \\
NEFA (mmol/l) & $1.64 \pm 0.11(7)$ & $1.30 \pm 0.11(9)$ \\
Triglycerides $(\mathrm{mmol} / \mathrm{l})$ & $1.56 \pm 0.18(8)$ & $1.44 \pm 0.14(9)$ \\
\hline
\end{tabular}

Data are means \pm SEM (number of mice in parentheses)

$* * p<0.01$ vs WT mice

Testosterone has been shown not only to increase lean mass, but also to decrease fat mass [28]. The serum concentrations of testosterone were increased in male a

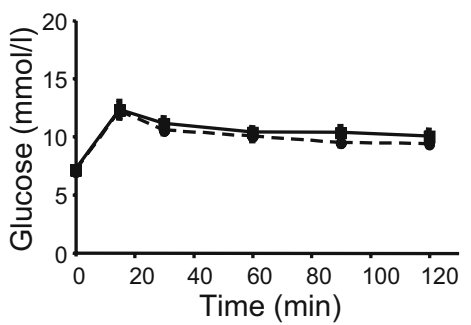

b

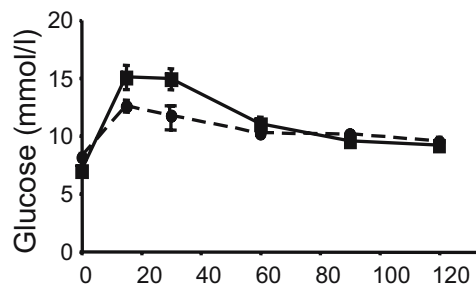

C

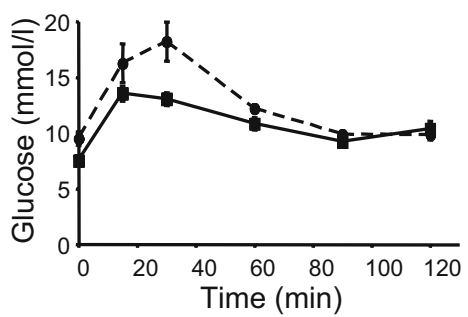

d

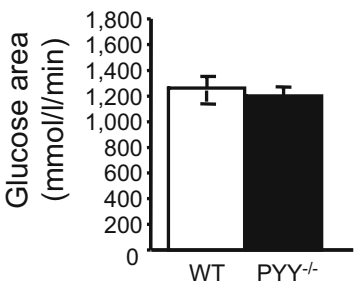

e

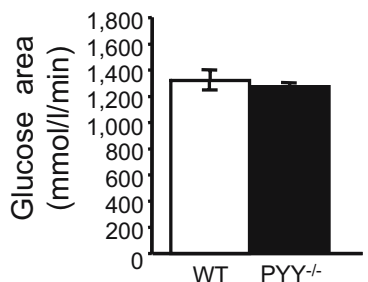

f

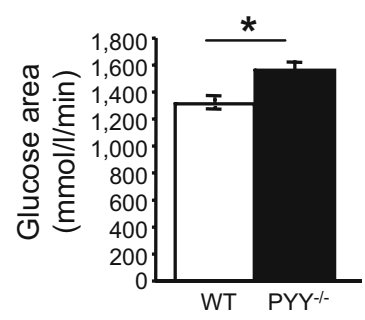

g

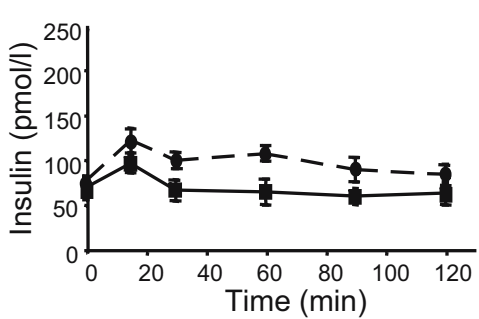

h

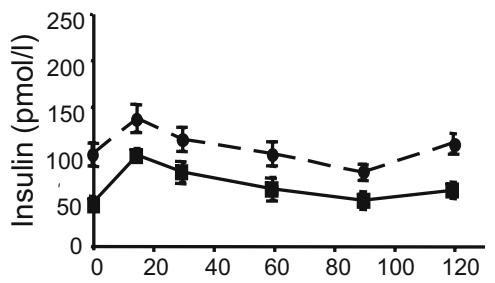

i

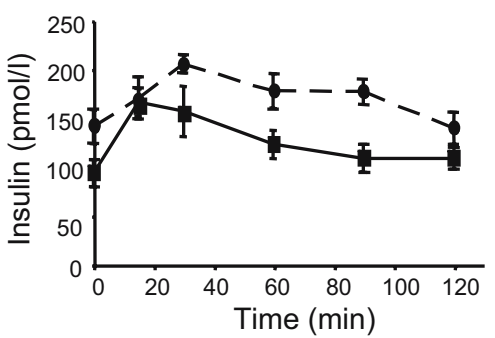

j

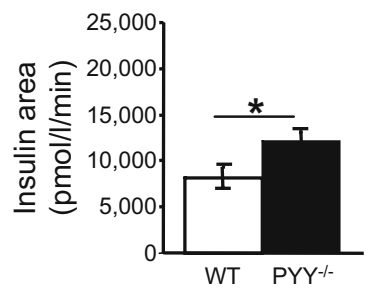

k

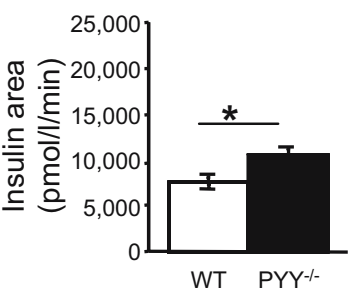

I

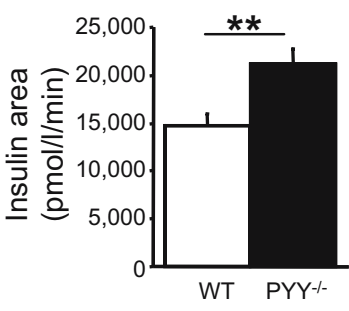

Fig. 4 Effect of PYY deficiency on glucose homeostasis. Changes in serum glucose levels after i.p. glucose injection $(1.0 \mathrm{mg} / \mathrm{kg})$ in $24-$ h-fasted chow-fed female (a) wild-type (filled squares) $(n=7)$ and $P y^{--}$(filled circles) $(n=8)$ mice and in (b) male wild-type (filled squares) $(n=6)$ and Pyy ${ }^{-1-}$ (filled circles) $(n=7)$ mice at 13 weeks of age. c Changes in serum glucose levels after i.p. glucose injection $(1.0 \mathrm{mg} / \mathrm{kg})$ in 24 -h-fasted high-fat-fed male wild-type (filled

squares) $(n=7)$ and $\mathrm{Pyy}^{--}$(filled circles) mice $(n=8)$ at 13 weeks of age. d-f Glucose response AUCs. $\mathbf{g}-\mathbf{i}$ Changes in insulin levels after i.p. glucose injection $(1.0 \mathrm{mg} / \mathrm{kg})$ in $24-\mathrm{h}$-fasted chow or highfat-fed female and male wild-type (filled squares) and $\mathrm{Pyy}^{-1-}$ (filled circles) mice. $\mathbf{j}-\mathbf{I}$ Insulin response AUCs. Data are means \pm SEM of the number of mice shown in parentheses. ${ }^{*} p<0.05$, ${ }^{*} * p<0.01$ vs the comparison shown by horizontal bars. WT, wild-type 
significant effect of Pyy deletion on serum glucagon concentrations in male or female animals (Table 1), consistent with the observation that our Pyy knock-out strategy did not alter the morphology or the number of glucagon-staining alpha cells in the islets of Langerhans (Fig. 2g,h).

As an index of hypothalamo-pituitary-thyroid function, an important determinant of resting metabolic rate [29], serum-free T4 levels, were measured in $P y y^{--}$and wildtype mice. There was no significant effect of Pyy ablation on serum-free T4 levels in mice of either gender or dietary condition (Tables 1 and 2).

Unaltered motor activity in $P y y^{-/}$mice

Mice were tested for general activity as well as for different motor activity tasks over a period of 14 days. Male and female $\mathrm{Pyy}^{---}$and wild-type mice at 14-16 weeks of age were indistinguishable in the amount and pattern of activity (Fig. 5). Furthermore, motor activity assessed in the openfield test was not different between male and female $P y y^{--}$ and wild-type mice (data not shown), suggesting that locomotion is not altered in these mice.

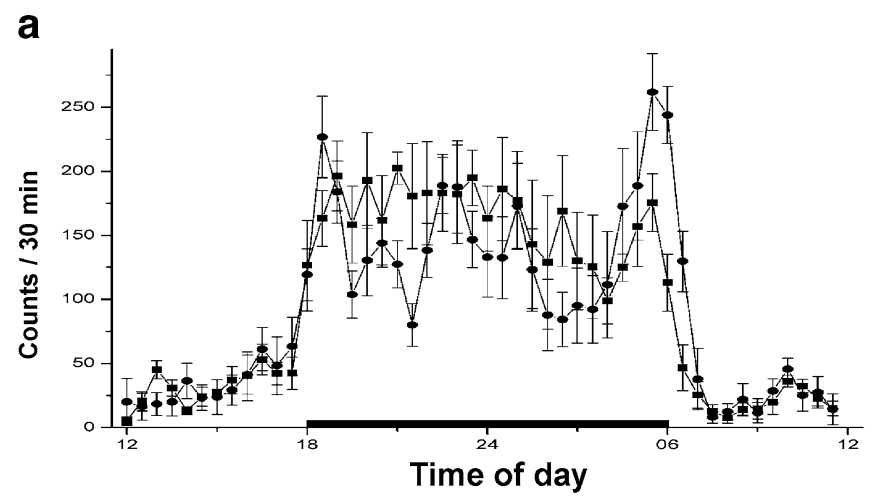

b

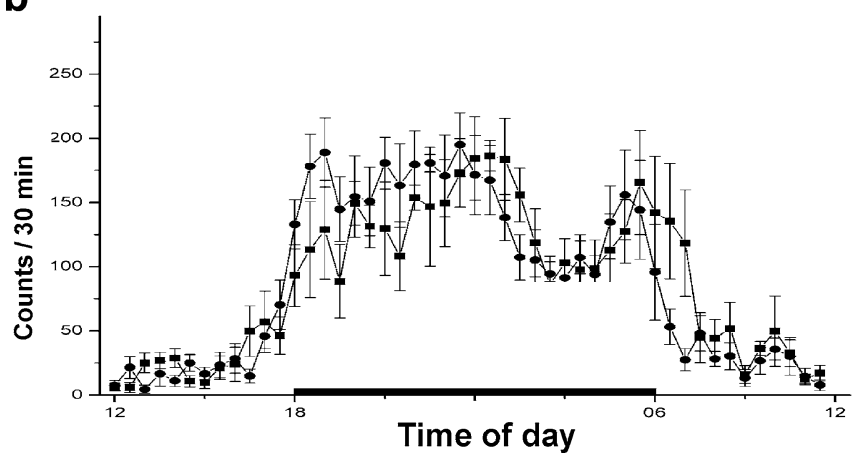

Fig. 5 Effect of PYY deficiency on motor activity: 24-h activity pattern of 16-week-old chow-fed female (a) and male (b) wild-type (filled squares) and Pyy ${ }^{--}$mice (filled circles). Dark bar, night phase. Data are means \pm SEM of eight mice per group
Hypothalamic changes in neuropeptide mRNA expression in $\mathrm{Pyy}^{--}$mice

To assess possible central mechanisms for changes in energy homeostasis in $\mathrm{Pyy}^{-/}$mice, we analysed the expression of hypothalamic neuropeptides known to regulate energy balance such as NPY, POMC and GHRH.

Fourteen-week-old female $P y y$ knock-outs did not show any significant changes in Npy mRNA expression in the arcuate nucleus of the hypothalamus, but exhibited a $51 \%$ increase in Pomc mRNA expression compared with controls (Fig. 6a-d), which was consistent with the significant reduction in food intake (Table 1) and fat mass (Fig. 3e) observed in these animals. Fourteen-weekold male $\mathrm{Pyy}^{-/-}$mice showed a significant $21 \%$ reduction in Npy and a significant $25 \%$ increase in Pomc mRNA expression levels in the arcuate nucleus compared with wild-type mice (Fig. 6e-h). While these changes in male mice were associated with increased lean mass and increased serum concentrations of IGF-I and testosterone, consistent with the expected effects of reduced central NPY-ergic expression [30], they were not associated with reductions in food intake.

The mRNA expression of the growth-promoting neuropeptide, Ghrh, was unaltered in the arcuate nucleus (Fig. 6i, j), but showed a significant $32 \%$ increase in the ventromedial hypothalamic nucleus of $\mathrm{Pyy}^{--}$mice (Fig. 6k,1), consistent with activation of the somatotrophic axis and the significantly higher serum levels of IGF-I (Table 1) and increased lean mass (Fig. 3f) in the chow-fed male $\mathrm{Pyy}^{-/}$mice.

\section{Discussion}

This study demonstrates that PYY-mediated pathways are important for the regulation of body composition, serum insulin levels and glucose homeostasis. Deficiency of Pyy resulted in late-onset obesity -indicated by significant increases in bodyweight and fat mass - in female mice on a chow diet. Male $\mathrm{Pyy}^{--}$mice were resistant to late-onset obesity on a chow diet, but became significantly fatter than wild-types when fed a high-fat diet. These effects of Pyy ablation were associated with significant increases in basal and/or glucose-induced serum insulin levels in both male and female $P y y^{-/-}$mice, in the absence of increases in basal or fasting-induced food intake. Moreover, these increases in serum insulin levels were clearly apparent in chow-fed $P y y^{-1-}$ mice at 13 weeks of age. Since hyperinsulinaemia is causally linked to the aetiology of obese states [23, 24], it is possible that hyperinsulinaemia contributes to the development of increased adiposity in $\mathrm{Pyy}^{-/-}$mice. The elevated basal and glucose-induced serum insulin levels seen in our $P y y^{-/}$mice are most probably due to the lack of direct PYY inhibitory action on Y1 receptors in the pancreas [31, 32], as well as indirectly via a lack of PYY stimulation of Y2 receptors in the brainstem, which is known to alter vagal output [33]. 
Females

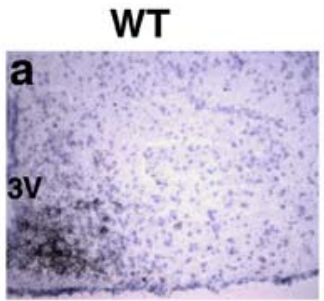

$100 \pm 4.3$

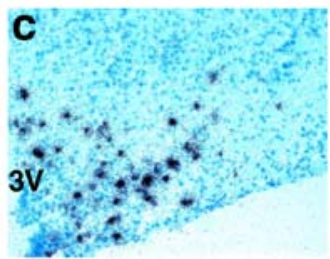

$100 \pm 1.95$

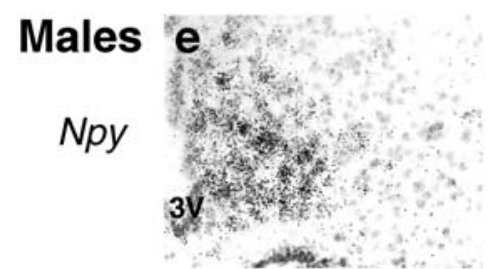

$100 \pm 3.03$
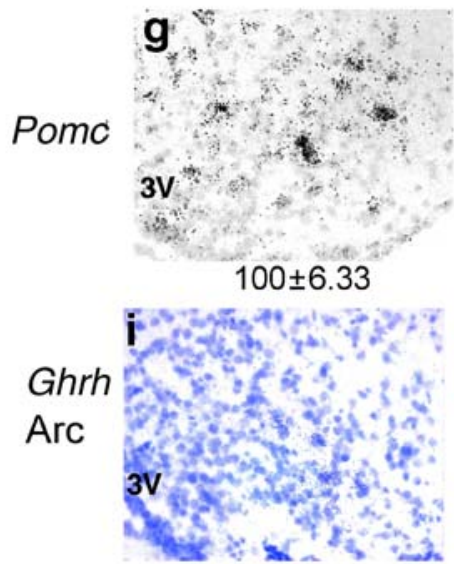

$100 \pm 6.78$

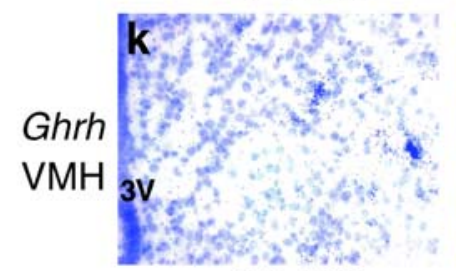

$100 \pm 5.28$

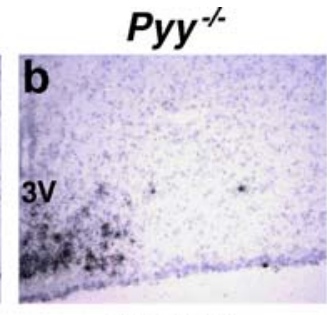

$93 \pm 12.7$

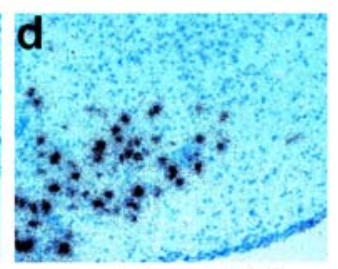

$151 \pm 9.42^{\star \star *}$

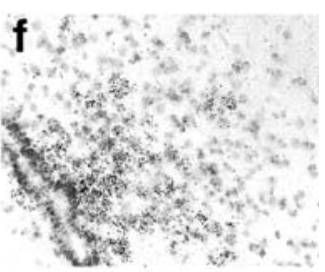

$79 \pm 4.04^{\star \star}$

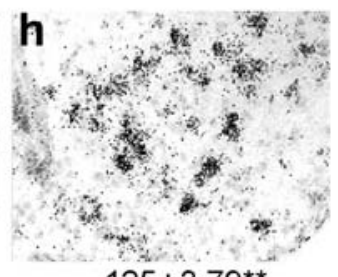

$125 \pm 3.79^{\star \star}$

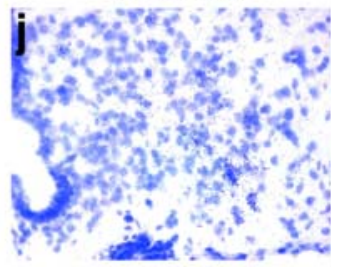

$109 \pm 5.08$

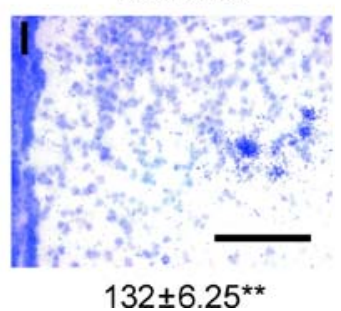

$132 \pm 6.25^{\star *}$

Fig. 6 Effect of PYY deletion on Npy, Pomc and Ghrh mRNA levels in the hypothalamus of 14-week-old female and male wildtype and $P y y^{--}$mice. High-power bright-field photomicrographs of dipped sections obtained from wild-type and $P y y^{-/}$mice after in situ hybridisation. Npy (a, b, e, f) in the arcuate hypothalamic nucleus (Arc); Pomc (c, d, $\mathbf{g}, \mathbf{h})$; Ghrh in the arcuate (i, j) and ventromedial hypothalamic $(V M H)$ nucleus $(\mathbf{k}, \mathbf{l})$. Scale bar $=10 \mu \mathrm{m}$. ${ }^{* *} p<0.01,{ }^{* * *} p<0.001$ vs wild-type mice. Pictures are representative of mRNA expression from five mice per group. $3 \mathrm{~V}$, 3rd ventricle

PYY and its processed form, PYY3-36, are known to activate Y-receptors in the arcuate nucleus of the hypothalamus and brainstem, thereby regulating the expression of NPY and POMC. Selective activation of Y2 receptors by PYY3-36 administration has been shown to downregulate $N P Y$ mRNA and upregulate $P O M C$ mRNA expression in the arcuate nucleus [12]. Interestingly, removal of the $P Y Y$ gene and therefore removal of the ability to activate Y1, Y2 or Y5 receptors either decreased or had no effect on the mRNA expression of NPY in the arcuate nucleus. Moreover, in both male and female mice, $P y y$ ablation resulted in a significant increase of Pomc expression in the hypothalamic arcuate nucleus. These changes in hypothalamic peptide expression may be caused by the high serum insulin levels observed in $\mathrm{Pyy}^{-/}$mice, since peripheral insulin administration is known to inhibit hypothalamic Npy and stimulate Pomc mRNA expression [34]. The observed changes in hypothalamic Pomc expression are in keeping with the significant reduction in food intake observed in the young (but not old) female mice. In male $P y y^{-1}$ mice, the increase in lean mass and resistance to late-onset obesity on a chow diet may be further attributed to stimulation of the hypothalamo-pituitary somatotrophic axis via increased Ghrh mRNA expression in the ventromedial hypothalamic nucleus and elevated IGF-I levels, as well as the increased serum testosterone levels $[28,35]$.

Recently, another report of a Pyy knock-out mouse was published [36]. In that publication, fasting-induced food intake and bodyweight of male and female chow-fed Pyy knock-out animals was no different from that of wild-types, albeit basal food intake, body composition, glucoseinduced glycaemia and insulinaemia, and ageing- and diet-induced obesity were not investigated. It is important to differentiate that PPY expression was also absent in the previously published $\mathrm{Pyy}^{--}$mouse model effectively making it a double Pyy/Ppy knock-out mouse.

In summary, we describe that the absence of PYY has a significant effect on body composition and on basal and glucose-induced serum insulin levels. Since circulating PYY levels are decreased in obese, hyperinsulinaemic people [37], our findings suggest that low circulating PYY concentrations may causally contribute to the development of hyperinsulinaemia and obesity, during ageing or under conditions of long-term consumption of a high-fat diet, respectively.

Acknowledgements $\mathrm{We}$ are grateful to $\mathrm{D}$. Withers and $\mathrm{R}$. Batterham (UCL, London, UK) for providing the mouse Pyy gene. We thank J. Ferguson (Garvan Institute) for veterinary advice and the staff of the Garvan Institute Biological Testing Facility. We thank D. James, T. Kraegen and L. Heilbronn (Garvan Institute) for critical review of the manuscript. This research was supported by the National Health and Medical Research Council (Project Grant \#230816), a Grant by the Prader Willi Foundation and NHMRC Fellowships to A. Sainsbury and H. Herzog. 


\section{References}

1. Larhammar D (1996) Evolution of neuropeptide Y, peptide YY and pancreatic polypeptide. Regul Pept 62:1-11

2. Herzog H (2003) Neuropeptide $Y$ and energy homeostasis: insights from Y receptor knockout models. Eur J Pharmacol 480:21-29

3. Pieribone VA, Brodin L, Friberg K et al (1992) Differential expression of mRNAs for neuropeptide Y-related peptides in rat nervous tissues: possible evolutionary conservation. J Neurosci 12:3361-3371

4. Lundberg JM, Terenius L, Hökfelt T, Tatemoto K (1984) Comparative immunohistochemical and biochemical analysis of pancreatic polypeptide-like peptides with special reference to presence of neuropeptide $\mathrm{Y}$ in central and peripheral neurons. J Neurosci 4:2376-2386

5. Blomqvist AG, Herzog H (1997) Y-receptor subtypes-how many more? Trends Neurosci 20:294-298

6. Adrian TE, Ferri GL, Bacarese-Hamilton AJ, Fuessl HS, Polak JM, Bloom SR (1985) Human distribution and release of a putative new gut hormone, peptide YY. Gastroenterology 89:1070-1077

7. Onaga T, Zabielski R, Kato S (2002) Multiple regulation of peptide YY secretion in the digestive tract. Peptides 23:279-290

8. Chelikani PK, Haver AC, Reidelberger RD (2005) Intravenous infusion of peptide $\mathrm{YY}(3-36)$ potently inhibits food intake in rats. Endocrinology 146:879-888

9. Riediger T, Bothe C, Becskei C, Lutz TA (2004) Peptide YY directly inhibits ghrelin-activated neurons of the arcuate nucleus and reverses fasting-induced c-Fos expression. Neuroendocrinology 79:317-326

10. Batterham RL, Cowley MA, Small CJ et al (2002) Gut hormone PYY3-36 physiologically inhibits food intake. Nature 418:650-654

11. Adams SH, Won WB, Schonhoff SE, Leiter AB, Paterniti JR Jr (2004) Effects of peptide YY[3-36] on short-term food intake in mice are not affected by prevailing plasma ghrelin levels. Endocrinology 145:4967-4975

12. Challis BG, Pinnock SB, Coll AP, Carter RN, Dickson SL, O'Rahilly S (2003) Acute effects of PYY3-36 on food intake and hypothalamic neuropeptide expression in the mouse. Biochem Biophys Res Commun 311:915-919

13. Halatchev IG, Ellacott KL, Fan W, Cone RD (2004) Peptide YY336 inhibits food intake in mice through a melanocortin-4-receptorindependent mechanism. Endocrinology 145:2585-2590

14. Pittner R, Moore C, Bhavsar S et al (2004) Effects of PYY[3$36]$ in rodent models of diabetes and obesity. Int J Obes Relat Metab Disord 28:963-971

15. Tschop M, Castaneda TR, Joost HG et al (2004) Physiology: does gut hormone PYY3-36 decrease food intake in rodents? Nature 430: $1 \mathrm{p}$ following 165; discussion 2 pp following 165

16. Kushi A, Sasai H, Koizumi H, Takeda N, Yokoyama M, Nakamura M (1998) Obesity and mild hyperinsulinemia found in neuropeptide Y-Y1 receptor-deficient mice. Proc Natl Acad Sci USA 95:15659-15664

17. Molero JC, Jensen TE, Withers PC et al (2004) c-Cbl-deficient mice have reduced adiposity, higher energy expenditure, and improved peripheral insulin action. J Clin Invest 114:1326-1333

18. Hoentjen F, Hopman WP, Jansen JB (2001) Effect of circulating peptide YY on gallbladder emptying in humans. Scand J Gastroenterol 36:1086-1091
19. Tatemoto $K$ (1982) Isolation and characterization of peptide YY (PYY), a candidate gut hormone that inhibits pancreatic exocrine secretion. Proc Natl Acad Sci USA 79:2514-2518

20. Bottcher G, Ahren B, Lundquist I, Sundler F (1989) Peptide YY: intrapancreatic localization and effects on insulin and glucagon secretion in the mouse. Pancreas 4:282-288

21. Karlsson S, Ahrén B (1996) A role for islet peptide YY in the regulation of insulin secretion. Acta Physiol Scand 157:305-306

22. van den Hoek AM, Heijboer AC, Corssmit EPM et al (2004) PYY3-36 reinforces insulin action on glucose disposal in mice fed a high-fat diet. Diabetes 53:1949-1952

23. Assimacopoulos-Jeannet F, Jeanrenaud B (1976) The hormonal and metabolic basis of experimental obesity. Clin Endocrinol Metab 5:337-365

24. Standridge M, Alemzadeh R, Zemel M, Koontz J, MoustaidMoussa N (2000) Diazoxide down-regulates leptin and lipid metabolizing enzymes in adipose tissue of Zucker rats. FASEB J 14:455-460

25. Furuse M, Mabayo RT, Okumura J (1997) Effects of dietary cholestyramine on the utilisation of diets containing medium or long chain triacylglycerols by chicks. Br Poult Sci 38:436-438

26. Sainsbury A, Schwarzer C, Couzens M et al (2002) Important role of hypothalamic $\mathrm{Y} 2$ receptors in bodyweight regulation revealed in conditional knockout mice. Proc Natl Acad Sci USA 99:8938-8943

27. West DB, Boozer CN, Moody DL (1992) Dietary obesity in nine inbred mouse strains. Am J Physiol 262:R1025-R1032

28. Mudali S, Dobs AS (2004) Effects of testosterone on body composition of the aging male. Mech Ageing Dev 125:297-304

29. al-Adsani H, Hoffer LJ, Silva JE (1997) Resting energy expenditure is sensitive to small dose changes in patients on chronic thyroid hormone replacement. J Clin Endocrinol Metab 82:1118-1125

30. Pierroz DD, Catzeflis C, Aebi AC, Rivier JE, Aubert ML (1996) Chronic administration of neuropeptide $Y$ into the lateral ventricle inhibits both the pituitary-testicular axis and growth hormone and insulin-like growth factor I secretion in intact adult male rats. Endocrinology 137:3-12

31. Cho YR, Kim CW (2004) Neuropeptide Y promotes beta-cell replication via extracellular signal-regulated kinase activation. Biochem Biophys Res Commun 314:773-780

32. Morgan DG, Kulkarni RN, Hurley JD et al (1998) Inhibition of glucose stimulated insulin secretion by neuropeptide $\mathrm{Y}$ is mediated via the Y1 receptor and inhibition of adenylyl cyclase in RIN 5AH rat insulinoma cells. Diabetologia 41:1482-1491

33. Chen CH, Rogers RC (1997) Peptide YY and the Y2 agonist PYY-(13-36) inhibit neurons of the dorsal motor nucleus of the vagus. Am J Physiol 273:R213-R218

34. Havel PJ, Hahn TM, Sindelar DK et al (2000) Effects of streptozotocin-induced diabetes and insulin treatment on the hypothalamic melanocortin system and muscle uncoupling protein 3 expression in rats. Diabetes 49:244-252

35. Ho KK, O’Sullivan AJ, Hoffman DM (1996) Metabolic actions of growth hormone in man. Endocr J 43:S57-S63

36. Schonhoff SE, Baggio L, Ratineau C et al (2005) Energy homeostasis and gastrointestinal endocrine differentiation do not require the anorectic hormone peptide YY. Mol Cell Biol 25:4189-4199

37. Batterham RL, Cohen MA, Ellis SM et al (2003) Inhibition of food intake in obese subjects by peptide YY3-36. N Engl J Med 349:941-948 